\title{
Object Manipulation under Hybrid Active/Passive Closure
}

\author{
Tetsuyou Watanabe \\ Dep. of Mech. Eng. \\ Yamaguchi University \\ Ube, 755-8611, Japan \\ te-watanabe@ieee.org
}

\author{
Kensuke Harada \\ National Institute of \\ Advanced Industrial \\ Science and Technology \\ Tsukuba, 305-8568, Japan \\ kensuke.harada@aist.go.jp
}

\author{
Zhongwei Jiang \\ Dep. of Mech. Eng. \\ Yamaguchi University \\ Ube, 755-8611, Japan \\ jiang@yamaguchi-u.ac.jp
}

\author{
Tsuneo Yoshikawa \\ Dep. of Mech. Eng. \\ Graduate school of Eng. \\ Kyoto University \\ Kyoto, 606-8501, Japan \\ yoshi@mech.kyoto-u.ac.jp
}

\begin{abstract}
In this paper, we discuss the manipulation of an object under hybrid active/passive closure. We show the orthogonality between the directions of active and passive force closures for general grasping systems. Based on the orthogonality, we decompose the dynamics of grasping system into the "active part" and the "passive part". By using the decomposition, we show that the grasped object can be manipulated only by considering the dynamics of the active part. We also consider how to determine the desired internal forces in order to satisfy frictional constraints during the manipulation. In order to verify the validity of our approach, some simulation results are shown.
\end{abstract}

Index Terms - Active/Passive Force Closure, Manipulation, Orthogonality, Grasping

\section{INTRODUCTION}

The force closure has been one of the important properties of grasping [1]. However, the force closure had been interpreted by two ways [2]. One is that " any arbitrary force and moment can be applied to a grasped object by fingers. "and the other is that " any arbitrary external force and moment exerted on a grasped object can be balanced without changing the pre-loaded joint torques and the motion of the object can be completely constrained."

The former concept corresponds to that fingers (limbs) can move a grasped object in arbitrary directions. On the other hand, the latter concept corresponds to the power grasps [3] where fingers can grasp object stably without changing the pre-loaded joint torques. In order to remove the ambiguity of the definition, Yoshikawa [4] named the former concept active force closure, and the latter concept passive force closure.

Here, we can encounter many cases where a grasping system has both the active and the passive closure properties. Yoshikawa [4] called such grasps as hybrid active/passive-closure grasps. For the hybrid active/passive closure properties included in many grasping systems, the key issue is the orthogonality between the "active" and the "passive" parts. While we introduced the orthogonality between them [5], the orthogonality has been proven for a limited class of grasping systems. Also, the control algorithm for manipulating an object has never been considered.

In this paper, we show the orthogonality between the active and the passive parts for general grasping systems. Also, we propose a control algorithm for manipulation of an object considering the hybrid active/passive closure properties for general grasping systems. While our algorithm can be applicable for general grasping systems, it has the following merit; When controlling the motion of the grasped object, our algorithm does not need to consider the whole dynamics of the grasping systems. This is due to the orthogonal property between the active and the passive parts of the grasping system.

Previously, hybrid position/force control has been proposed for several robot manipulators [6]. Our proposed algorithm can include the conventional hybrid control algorithm. However, the extension is not straightforward. Different from the robot manipulators, a grasping system has uncontrollable parts such as the passive part. Our proposed control algorithm can deal with such uncontrollable parts. Off course, the frictional constraints, which are not considered in the conventional hybrid control, also can be dealt with.

This paper is organized as follows. At first, the target system is shown and the directions of active and passive force closures are defined. Then, we show the directions of active force closure are orthogonal to those of passive force closure. Using the orthogonality, we derive a control algorithm. We also discuss how to determine the desired internal forces for the manipulation. Lastly, we show some simulation results to show the validity of our approach.

\section{A. Related Works}

Firstly Trinkle [2] gave force closure two interpretations as described above. Yoshikawa [4] named the two concepts active and passive force closures. Previously, the active and the passive force closures have been studied separately.

As for active force closure, $\mathrm{Li}$ et al. [7], Cole et al. [8], and Yokokohji et al. [9] presented control algorithms for the case of point contact, rolling contact, and softfinger contact, respectively, for the manipulation of an object grasped by fingertips. Harada et al. analyzed the active force closure [10] and presented a control algorithm [11] for manipulating multiple objects. There are some researches focusing on active force closure in the general grasping systems. Trinkle et al. [12] discussed a grasp planning for manipulating an enveloped object with sliding contacts in 2 dimensional space. Bicchi et al. [13] analyzed the manipulability of the general grasping systems. Harada et al. [14] presented a sufficient condition for the manipulation of Envelope Family and realize the manipulation 
with constant joint driving torques. Park et al. [15] derived contact forces and accelerations consistent with dynamics and friction law for a given torque-wrench pair.

Note that there exist two ways as which we can regard active force closure. One is that fingers (limbs) can move a grasped object in arbitrary directions as described above. The other is that active force closure is standard notation of force closure and is necessary but not sufficient for passive force closure [16]. We follow the precedent active force closure by Yoshikawa [4] by adopting the following definition.

Active force closure: A grasp is said to be of active force closure if any resultant force and moment can be applied to the object by fingers and the maintenance of the object's equilibrium requires the application of the resultant force and moment applied by the fingers.

On the other hand, the passive force closure (power grasp) have been researched with respect to the analysis of robustness, the analysis of indeterminate forces, the formulation of contact force distribution, the optimization of pre-loaded joint torques, and so on [16]-[24].

There are many cases where a grasping system has both active and passive force-closure properties simultaneously. Only recently, the authors have begun some researches focusing on the both properties [5], [25]. However, the obtained results are still limited. In this paper, we show the orthogonality between the active and the passive parts for general grasping systems, and present a control algorithm for the manipulation, based on the orthogonality.

\section{NOMENCLATURE}

The following nomenclatures are used in this paper. $N \quad$ Number of fingers.

$M_{i} \quad$ Number of joints of the $i$ th finger $(i=$ $1,2, \cdots, N)$.

$L_{i} \quad$ Number of contact points on the $i$ th finger.

$M \quad$ Number of total joints $\left(=\sum_{i=1}^{N} M_{i}\right)$.

$L \quad$ Number of total contact points $\left(=\sum_{i=1}^{N} L_{i}\right)$.

D $\quad 3 / 6$ in $2 / 3$ dimensional space.

$d \quad 2 / 3$ in $2 / 3$ dimensional space.

$\Sigma_{R} \quad$ Reference coordinate frame.

$\Sigma_{O} \quad$ Object coordinate frame fixed at the object.

$\Sigma_{G} \quad$ Coordinate frame fixed at the center of gravity of the object.

$C_{i j} \quad$ The $j$ th contact point of the $i$ th finger $(j=$ $\left.1,2, \cdots, L_{i}\right)$.

$\Sigma_{C_{i j}} \quad$ Coordinate frame fixed at $C_{i j}$.

$\Sigma_{F_{i j}} \quad$ Coordinate frame fixed at the link of the $i$ th finger, on which there exists $C_{i j}$.

$\boldsymbol{q}_{i} \in \mathcal{R}^{M_{i}} \quad$ Joint angle vector of the $i$ th finger

$p_{I} \in \mathcal{R}^{d}$ Position of the origin of $\Sigma_{I} \quad(I=$ $\left.O, G, C_{i j}, F_{i j}\right)$.

$\omega_{1} \in \mathcal{R}^{D-d}$ Rotational velocity vector of $\Sigma_{I}$.

$\nu_{C_{i j}} \in \mathcal{R}^{d}$ Contact point velocity at $C_{i j}$ where the component of contact point motion due to rolling is excluded ( $\nu_{C_{i j}}=\dot{p}_{C_{i j}}$ where rolling doesn't occur).

$v_{I} \in \mathcal{R}^{D} \quad\left(\dot{p}_{I}^{T} \omega_{I}^{T}\right)^{T}$

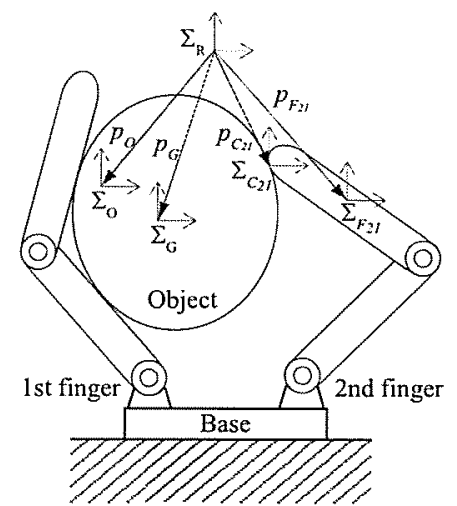

Fig. 1. Target System $(N=2)$

$w_{I} \in \mathcal{R}^{D}$ Resultant force and moment applied to the object at $\Sigma_{I}(I=G, O)$.

$f_{i j} \in \mathcal{R}^{d}$ Contact force vector at $C_{i j}$.

$f \in \mathcal{R}^{L d}\left(f_{11}^{T} f_{12}^{T} \cdots f_{N L_{N}}^{T}\right)^{T}$.

$\tau_{c} \in \mathcal{R}^{M}$ Joint torque vector equivalent to $f$.

$n_{f_{i j}} \quad$ Normal force component of $f_{i j}$.

$t_{f_{i j}, k} \quad$ Tangential force components of $f_{i j}(k=1,2)$.

$\mu_{i j} \quad$ Frictional coefficient at $C_{i j}$.

Note that since there exist some cases where it is convenient if the origin of $\Sigma_{O}$ doesn't coincide with the center of gravity of the object, we define not only $\Sigma_{O}$ but also $\Sigma_{G}$.

\section{TARGET SYSTEM AND DEFINITION}

\section{A. Target System}

The target system is shown in Fig.1. In this paper, we consider the case where an arbitrary shaped rigid object is grasped by $N$ fingers of a robotic hand. We make the following assumptions: 1) Each finger makes a frictional point contact with the object, and sliding doesn't occur at each contact point. 2) There exists at most one contact point on each link of the fingers. 3) The number of contact points does not change and the remove of contact points does not occur.

\section{B. Definition}

When manipulating an object under hybrid active/passive closure, the directions of object motion is limited to the directions satisfying active force closure. To obtain a controller for manipulating an object, we decompose the dynamics of grasping system into the "active part" and the "passive part". Here, we do not control the object motion within the directions satisfying passive force closure since wrench applied on the object is counteracted without any additional joint torque input. For such purpose, we introduce the following two spaces within the generalized velocity space and the generalized force space (wrench space) of the object.

Space of Active Force Closure (SAFC); Let us consider the direction in which a grasped object can move 
within a constraint applied by fingers. If the fingers can do positive work on the object in that direction, we call the direction as direction of active force closure (DAFC). Also, we call the space, spanned by a set of the all DAFC's, as space of active force closure (SAFC).

Space of Passive Force Closure (SPFC); We call the direction, in which external force or moment can be counteracted without changing the joint torques, as direction of passive force closure (DPFC). Also, we call the space, spanned by a set of the all DPFC's, as space of passive force closure (SPFC).

\section{BASIC FORMULATION OF THE SYSTEM}

We discuss 3 dimensional case, but the formulation can be easily extended to 2 dimensional case.

\section{A. Kinematics of the system}

The relation between $v_{O}$ and $v_{G}$ is given by

$$
\boldsymbol{v}_{G}=\boldsymbol{D}_{G O} \boldsymbol{v}_{o}
$$

where

$$
D_{G O}=\left(\begin{array}{cc}
I & -\left[\left(p_{G}-p_{o}\right) \times\right] \\
O & I
\end{array}\right) \in \mathcal{R}^{D \times D}
$$

where $I$ represents an identity matrix, $O$ represents a zero matrix, $[a \times]$ represents a skew symmetric matrix equivalent to the cross product operation ( $[a \times] b=a \times b)$.

The relation between $\boldsymbol{v}_{F_{i j}}$ and $\dot{\boldsymbol{q}}_{i}$, and the relation among $\nu_{C_{i j}}, v_{F_{i j}}$ and $v_{o}$, respectively, are given as follows;

$$
v_{F_{i j}}=J_{F_{i j}} \dot{q}_{i}, \quad G_{O_{i j}}^{T} v_{O}=\nu_{C_{i j}}=D_{F_{i j}}^{T} v_{F_{i j}}
$$

where $J_{F_{i j}} \in \mathcal{R}^{D \times M_{i}}$ denotes the Jacobian matrix and

$$
\begin{aligned}
\boldsymbol{G}_{O_{i j}} & =\left(\left[\left(p_{C_{i j}}-p_{o}\right) \times\right]\right) \in \mathcal{R}^{D \times d}, \\
D_{F_{i j}} & =\left(\left[\begin{array}{c}
I \\
\left.\left[\left(p_{C_{i j}}-p_{F_{i j}}\right) \times\right]\right) \in \mathcal{R}^{D \times d} .
\end{array}\right.\right.
\end{aligned}
$$

By using the following vectors and matrices,

$$
\begin{aligned}
& \dot{\boldsymbol{q}}=\left(\begin{array}{cccc}
\dot{\boldsymbol{q}}_{1}^{T} & \dot{\boldsymbol{q}}_{2}^{T} & \cdots & \dot{\boldsymbol{q}}_{N}^{T}
\end{array}\right)^{T} \in \mathcal{R}^{M}, \\
& \boldsymbol{J}=\operatorname{diag}\left(\left(\begin{array}{c}
\boldsymbol{D}_{F_{11}}^{T} \boldsymbol{J}_{F_{11}} \\
\vdots \\
\boldsymbol{D}_{F_{1 L_{1}}}^{T} \boldsymbol{J}_{F_{1 L_{1}}}
\end{array}\right) \quad\left(\begin{array}{c}
\boldsymbol{D}_{F_{21}}^{T} \boldsymbol{J}_{F_{21}} \\
\vdots \\
\boldsymbol{D}_{F_{2 L_{2}}}^{T} \boldsymbol{J}_{F_{2 L_{2}}}
\end{array}\right)\right. \\
& \left.\cdots\left(\begin{array}{c}
D_{F_{N 1}}^{T} J_{F_{N 1}} \\
\vdots \\
D_{F_{N L}}^{T} J_{F_{N L}}
\end{array}\right)\right) \in \mathcal{R}^{L d \times M}, \\
& G_{O}=\left(\begin{array}{llll}
G_{O_{11}} & G_{O_{12}} & \cdots & G_{O_{N L}}
\end{array}\right) \in \mathcal{R}^{D \times L d}, \\
& A_{O}=\left(\begin{array}{ll}
J & -G_{O}^{T}
\end{array}\right) \in \mathcal{R}^{L d \times(M+D)}
\end{aligned}
$$

where diag denotes a block diagonal matrix, from (2) we obtain

$$
\boldsymbol{A}_{O}\left(\begin{array}{cc}
\dot{\boldsymbol{q}}^{T} & \boldsymbol{v}_{o}^{T}
\end{array}\right)^{T}=\boldsymbol{o}
$$

where $o$ denotes a zero vector. By solving (3), we obtain

$$
\left(\begin{array}{c}
\dot{q} \\
v_{o}
\end{array}\right)=E_{P}^{T} \dot{\zeta}=\left(\begin{array}{c}
E_{P 1}^{T} \\
E_{P 2}^{T}
\end{array}\right) \dot{\zeta}
$$

where $E_{P} \in \mathcal{R}^{a \times(M+D)}$ denotes an orthogonal matrix whose rows form bases of the null space of $A_{O}, \dot{\zeta} \in \mathcal{R}^{a}$ is an arbitrary vector expressing the magnitude of each column of $\boldsymbol{E}_{P}^{T}$. Note that $\dot{\boldsymbol{\zeta}}$ represent the object motion within the constraint applied by the fingers, and can be selected arbitrary.

\section{B. Statics of the system}

From (1) and the principle of virtual work, the relation between $w_{O}$ and $w_{G}$ is given by

$$
w_{o}=D_{G O}^{T} w_{G}
$$

From (3) and the principle of virtual work, the relation among $f, w_{o}$ and $\tau_{c}$ is given by

$$
\left(\begin{array}{cc}
\tau_{c}^{T} & -\boldsymbol{w}_{o}^{T}
\end{array}\right)^{T}=\boldsymbol{A}_{O}^{T} \boldsymbol{f}=\left(\begin{array}{ll}
J & -G_{O}^{T}
\end{array}\right)^{T} f .
$$

From (6), the following relation is obtained;

$$
f=\left(J^{T}\right)^{+} \tau_{c}+\left(I-\left(J^{T}\right)^{+} J^{T}\right) \tilde{k}_{1},
$$

where $\left(\boldsymbol{J}^{T}\right)^{+}$denotes the pseudo-inverse matrix of $J^{T}$ and $\tilde{k}_{1} \in \mathcal{R}^{L d}$ denotes an arbitrary vector. Note that the force of the second term in the right side of (7) expresses an internal force which makes no influence on the joint torques.

By substituting (7) into (6), we get

$$
\begin{aligned}
\left(\begin{array}{c}
\tau_{c} \\
-w_{o}
\end{array}\right) & =\left(\begin{array}{c}
I \\
-G_{O}\left(J^{T}\right)+
\end{array}\right) \tau_{c}+\left(\begin{array}{c}
O \\
-G_{O}\left(I-\left(J^{T}\right)^{+} J^{T}\right)
\end{array}\right) \tilde{k}_{1} \\
& \triangleq\left(\begin{array}{c}
I \\
-G_{O J}
\end{array}\right) \tau_{c}+\left(\begin{array}{c}
O \\
-\Xi
\end{array}\right) k_{1}
\end{aligned}
$$

where $G_{O J}=G_{O}\left(J^{T}\right)^{+}, \Xi \in \mathcal{R}^{D \times p}$ is an orthogonal matrix whose columns form bases of the $G_{O}\left(I-\left(J^{T}\right)^{+} J^{T}\right)$, and $k_{1} \in \mathcal{R}^{p}$ is an arbitrary vector expressing the magnitude of each column of $\Xi$. Note that the second term in the right side of (8) can express a generable resultant force without changing the joint torques.

\section{Frictional constraints}

The frictional constraint at $C_{i j}(i=1,2, \cdots, N, j=$ $1,2, \cdots, L_{i}$ ) can be represented by

$$
\mathcal{F}_{f i j}=\left\{f_{i j} \mid \sqrt{t_{f_{i j}, 1}^{2}+t_{f_{i j}, 2}^{2}} \leq \mu_{i j} n_{f_{i j}}, n_{f_{i j}} \geq 0\right\} .
$$

Aggregating (9) for all contact points, we obtain

$$
\mathcal{F}_{f}=\left\{f \mid f_{i j} \in \mathcal{F}_{f i j}, \forall f_{i j}\right\}
$$




\section{Dynamics of the object and the fingers}

The equation of motion of the object and the fingers, respectively, can be represented as follows;

$$
\begin{aligned}
M_{r} \dot{v}_{G}+h_{r} & =w_{G}=D_{G O}^{-T} w_{O}, \\
M_{q} \ddot{q}+h_{q}+\tau_{c} & =\tau
\end{aligned}
$$

where $\tau$ denotes the joint driving torques, $M_{r}$ and $M_{q}$ are the inertia tensors of the object and the fingers, respectively, $h_{r}$ and $h_{q}$ are the terms representing centrifugal, Coriolis and gravitational forces of the object and the fingers, respectively.

From (1) and (5), (11) can be rewritten by

$$
M_{O r} \dot{v}_{o}+h_{O r}=w_{O}=G_{O} f
$$

where

$$
\begin{aligned}
M_{O r} & =D_{G O}^{T} M_{r} D_{G O} \\
h_{O r} & =D_{G O}^{T} M_{r} \dot{D}_{G O} v_{o}+D_{G O}^{T} h_{r} .
\end{aligned}
$$

\section{ORTHOGONALITY BETWEEN SAFC AND SPFC}

In this section, we show the orthogonality between SAFC and SPFC [5].

Let us consider the case where the object is stably grasped with $\tau_{c}$ and is in steady state. Namely, from (8) and $(10), \tau_{c}$ hold

$$
\left(\begin{array}{c}
\tau_{c} \\
-w_{O_{s t}}
\end{array}\right)=A_{O}^{T} f_{s t}=\left(\begin{array}{c}
I \\
-G_{O J}
\end{array}\right) \tau_{c}+\left(\begin{array}{c}
O \\
-\Xi
\end{array}\right) k_{1_{s t}}, f_{s t} \in \mathcal{F}_{f}
$$

where $w_{O_{s t}}, f_{s t}$ and $k_{1_{s t}}$, respectively, are $w_{O}, f$ and $k_{1}$ in the case where the system is in the steady state. SAFC and SPFC are considered in this case.

\section{A. $S A F C$}

At first, we consider a canonical/reduced form of $\boldsymbol{E}_{P 2}$. $E_{P}$ given in (4) can be written as follows (refer to [13]);

$$
\left(\begin{array}{c}
E_{P 1}^{T} \\
E_{P 2}^{T}
\end{array}\right)=\left(\begin{array}{ccc}
E_{P 11}^{T} & E_{P 12}^{T} & O \\
O & E_{P 22}^{T} & E_{P 23}^{T}
\end{array}\right)
$$

Here $\operatorname{Im}\left(\boldsymbol{E}_{P 11}^{T}\right)(=\operatorname{ker}(\boldsymbol{J}))$ denotes the redundancy subspace of joint velocities that do not affect the object velocities, and $\operatorname{Im}\left(\boldsymbol{E}_{P 23}^{T}\right)\left(=\operatorname{ker}\left(\boldsymbol{G}_{O}^{T}\right)\right)$ denotes the indeterminacy subspace of object velocities. Then, the directions indicated by the columns of $\left(\boldsymbol{E}_{P 22}^{T} \boldsymbol{E}_{P 23}^{T}\right)$ is the directions in which the object can move within the constraint applied by the fingers. Here, we consider the following transformation;

$$
\begin{aligned}
& \left(\begin{array}{c}
E_{P 1}^{T} \\
E_{P 2}^{T}
\end{array}\right) \Psi=\left(\begin{array}{ccc}
E_{P 11}^{T} & E_{P 12}^{T} & O \\
O & E_{P 22}^{T} & E_{P 23}^{T}
\end{array}\right)\left(\begin{array}{ccc}
I & O & O \\
O & \Psi_{2} & O \\
O & O & \Psi_{3}
\end{array}\right) \\
& \triangleq\left(\begin{array}{cc}
\boldsymbol{E}_{P 11}^{T} & \tilde{\boldsymbol{E}}_{P 1}^{T} \\
\boldsymbol{O} & \tilde{\boldsymbol{E}}_{P 2}^{T}
\end{array}\right) \\
& \dot{\zeta}=\Psi\left(\dot{\hat{\zeta}}^{T} \dot{\zeta}^{T}\right)^{T} \text {, }
\end{aligned}
$$

where $\Psi \in \mathcal{R}^{a \times a}$ is a nonsingular matrix for the transformation which makes both $\boldsymbol{E}_{P 22}^{T} \Psi_{2}$ and $\boldsymbol{E}_{P 23}^{T} \boldsymbol{\Psi}_{3}$ be orthogonal matrices. Note that since $\dot{\zeta}$ is arbitrary, $\dot{\hat{\zeta}} \in \mathcal{R}^{a-\tilde{a}}$ and $\dot{\bar{\zeta}} \in \mathcal{R}^{\tilde{a}}$ are also arbitrarily and express the magnitude of each column of $E_{P 11}^{T}$, and that of $E_{P 22}^{T} \Psi_{2}\left(E_{P 12}^{T} \Psi_{2}\right)$ and $\boldsymbol{E}_{P 23}^{T} \Psi_{3}$, respectively. Note also that $\tilde{E}_{P 1}^{T} \in \mathcal{R}^{M \times \bar{\alpha}}$ and $\tilde{E}_{P 2}^{T} \in \mathcal{R}^{D \times \bar{a}}$.

From the transformation, we obtain

$$
\left(\begin{array}{c}
E_{P 1}^{T} \\
E_{P 2}^{T}
\end{array}\right) \dot{\zeta}=\left(\begin{array}{cc}
E_{P 11}^{T} & \tilde{E}_{P 1}^{T} \\
O & \tilde{E}_{P 2}^{T}
\end{array}\right)\left(\begin{array}{c}
\dot{\hat{\zeta}} \\
\dot{\zeta}
\end{array}\right) .
$$

Note that $\operatorname{Im}\left(\tilde{\boldsymbol{E}}_{P 1}^{T}\right) \in \operatorname{Im}\left(\boldsymbol{J}^{T}\right)$ because $\operatorname{Im}\left(\boldsymbol{E}_{P 1}^{T}\right) \cap \operatorname{ker}(\boldsymbol{J})$ $=\operatorname{Im}\left(\boldsymbol{E}_{P 11}^{T}\right)$.

Then, the allowable motions of the object are given by

$$
\mathcal{A}=\left\{\Delta r \mid \Delta r=\tilde{E}_{P 2}^{T} \Delta \bar{\zeta}\right\}
$$

where $\Delta \boldsymbol{r}$ denotes the displacements of the object.

From (8), the work done by $\tau_{c}$ is given by

$$
\text { Work }=\Delta \boldsymbol{r}^{T} \boldsymbol{w}_{\mathrm{O}}=\Delta \boldsymbol{r}^{T} \boldsymbol{G}_{O . J} \tau_{c}+\Delta \boldsymbol{r}^{T} \boldsymbol{\Xi} \boldsymbol{k}_{1} \text {. }
$$

Note that $\Xi k_{1}$ is resultant force and moment which work to counteract external force and moment in the direction contained in $\operatorname{Im}(\boldsymbol{\Xi})$, only when the external force and moment are exerted on the object. Then, the applied force and moment by $\tau_{c}$ in the direction contained in $\left(\operatorname{Im}\left(G_{O J}\right) \cap \operatorname{Im}(\Xi)\right)$ will be counteracted. Then, defining $\boldsymbol{E}$ so that $(\boldsymbol{E} \boldsymbol{\Xi}) \in \mathcal{R}^{D \times D}$ is an orthogonal matrix, from (14) and (19), DAFC can be described as follows;

$$
\begin{array}{r}
\mathrm{DAFC}=\left\{\Delta r \mid \text { Work }=\Delta \boldsymbol{r}^{T} \boldsymbol{E} \boldsymbol{E}^{T} \boldsymbol{G}_{O J} \boldsymbol{\tau}_{c}>0, \Delta r \in \mathcal{A},\right. \\
\left(\boldsymbol{I}-\boldsymbol{E} \boldsymbol{E}^{T}\right) \boldsymbol{G}_{O J} \boldsymbol{\tau}_{c}+\boldsymbol{G}_{O}\left(\boldsymbol{I}-\left(\boldsymbol{J}^{T}\right)^{+} \boldsymbol{J}^{T}\right) \tilde{\boldsymbol{k}}_{1}=\boldsymbol{o}, \\
\left.\left(\boldsymbol{J}^{T}\right)^{+} \boldsymbol{\tau}_{c}+\left(\boldsymbol{I}-\left(\boldsymbol{J}^{T}\right)^{+} \boldsymbol{J}^{T}\right) \tilde{\boldsymbol{k}}_{1}+\boldsymbol{f}_{s t} \in \mathcal{F}_{f}\right\} .(21)
\end{array}
$$

When any $\left(J^{T}\right)^{+} \tau_{c}$, whose magnitude is finite, can be applied to the object for the appropriately assigned $f_{s t}$, (21) becomes

$$
\mathrm{DAFC}=\left\{\Delta \boldsymbol{r} \mid \Delta \boldsymbol{r}=\tilde{\boldsymbol{E}}_{P 2}^{T} \tilde{\boldsymbol{E}}_{P 2} \boldsymbol{E} \boldsymbol{E}^{T} \boldsymbol{G}_{O J} \boldsymbol{\tau}_{c}\right\} .
$$

\section{B. $S P F C$}

SPFC is the space spanned by the generable resultant forces and moments without changing $\tau_{c}$ at the steady state. From (14), such resultant forces and moments, $w_{O_{e x}}$ hold

$$
\begin{aligned}
& \left(\tau_{c}^{T}-w_{O_{e x}}^{T}-w_{O_{s t}}^{T}\right)^{T}=A_{O}^{T}\left(f_{s t}+f_{e x}\right)= \\
& \left(\begin{array}{ll}
I & -G_{O J}
\end{array}\right)^{T} \tau_{c}+\left(\begin{array}{ll}
O & -\Xi^{T}
\end{array}\right)^{T}\left(k_{1_{s t}}+k_{1_{e x}}\right)
\end{aligned}
$$

where $f_{c x}$ and $k_{1_{e x}}$, respectively, are $f$ and $k_{1}$, which correspond to $w_{O_{e x}}$. Note that

$$
\begin{aligned}
\left(\begin{array}{l}
\boldsymbol{o}-\boldsymbol{w}_{o_{e x}}^{T} \\
\boldsymbol{f}_{e x}
\end{array}\right)^{T}=\left(\boldsymbol{I}-\left(\boldsymbol{A}_{O}^{T} f_{e x}=\left(\begin{array}{ll}
\boldsymbol{O} & -\boldsymbol{\Xi}^{T}
\end{array}\right)^{T} \boldsymbol{k}_{1_{e x}}, \tilde{k}_{1_{e x}}\right.\right.
\end{aligned}
$$

If $f_{s t}+f_{e x} \in \mathcal{F}_{f}, \Xi k_{1_{e x}}$ becomes DPFC. Then, SPFC is given by

$$
\begin{gathered}
\mathrm{SPFC}=\left\{\boldsymbol{w} \mid \boldsymbol{w}=\boldsymbol{\Xi} k_{1_{e x}}, f_{e x}=\left(I-\left(J^{T}\right)^{+} J^{T}\right) \vec{k}_{1_{e x}},\right. \\
\left.f_{s t}+f_{e x} \in \mathcal{F}_{f}\right\}
\end{gathered}
$$




\section{Orthogonality}

In order to show the orthogonality between SAFC and SPFC, we have only to show that every possible DPFC is orthogonal to every possible DAFC. Then, let us consider the case where every column of $\tilde{\boldsymbol{E}}_{P 2}^{T}$ in (22) (or (21)) corresponds to DAFC and every column of $\Xi$ in (26) corresponds to DPFC.

From the definition of $\boldsymbol{E}_{P}$ (see (4)), $\boldsymbol{A}_{O} \boldsymbol{E}_{P}^{T}=\boldsymbol{O}$. Then, we obtain

$$
\boldsymbol{E}_{P} \boldsymbol{A}_{O}^{T} \boldsymbol{f}=\boldsymbol{o}, \quad \forall f
$$

Then, with respect to $f_{e x}$ in (25), we obtain

$$
E_{P} A_{O}^{T} f_{e x}=o, E_{P} A_{O}^{T}\left(I-\left(J^{T}\right)^{+} J^{T}\right) \tilde{k}_{1_{e x}}=o .
$$

Then, from (24), we obtain

$$
\boldsymbol{E}_{P}\left(\begin{array}{ll}
\boldsymbol{O} & -\boldsymbol{\Xi}^{T}
\end{array}\right)^{T} k_{1_{e x}}=\boldsymbol{o} .
$$

Since $k_{1_{e x}}$ is an arbitrary vector, we obtain

$$
\begin{aligned}
\boldsymbol{E}_{P}\left(\begin{array}{ll}
\boldsymbol{O}-\boldsymbol{\Xi}^{T}
\end{array}\right)^{T} & =\boldsymbol{O}, \boldsymbol{E}_{P 2} \boldsymbol{\Xi}=\boldsymbol{O} \\
\overline{\boldsymbol{E}}_{P 2} \boldsymbol{\Xi} & =\boldsymbol{O} .
\end{aligned}
$$

(27) represents the orthogonality between SAFC (DAFC) and SPFC (DPFC).

Remark: Let us consider the generalized force space of object, spanned by the all columns of $\Xi$ and $\tilde{E}_{P 2}^{T}$. Using $\Xi$, the generalized force space of object can be represented by

$$
\begin{aligned}
\left\{\boldsymbol{w}_{o} \mid \boldsymbol{w}_{O} \in \mathcal{R}^{D}\right\}=\operatorname{Im}(\boldsymbol{\Xi}) \oplus \operatorname{ker}\left(\boldsymbol{\Xi}^{T}\right) \\
\quad=\operatorname{Im}(\boldsymbol{\Xi}) \oplus \operatorname{ker}\left(\left(I-\left(\boldsymbol{J}^{T}\right)^{+} \boldsymbol{J}^{T}\right) \boldsymbol{G}_{O}^{T}\right) .
\end{aligned}
$$

$\operatorname{ker}\left(\left(I-\left(J^{T}\right)^{+} J^{T}\right) G_{O}^{T}\right)$ is given by

$$
\begin{aligned}
\operatorname{ker} & \left(\left(\boldsymbol{I}-\left(\boldsymbol{J}^{T}\right)^{+} \boldsymbol{J}^{T}\right) G_{O}^{T}\right) \\
\quad & =\operatorname{ker}\left(\boldsymbol{G}_{O}^{T}\right) \cup\left\{\boldsymbol{w}_{O} \mid \boldsymbol{G}_{O}^{T} \boldsymbol{w}_{O} \in \operatorname{Im}(\boldsymbol{J})\right\} \\
& =\left\{\boldsymbol{w}_{O} \mid\left(\boldsymbol{x}^{T} \boldsymbol{w}_{O}^{T}\right)^{T} \in \operatorname{ker}\left(\boldsymbol{A}_{O}\right), \boldsymbol{x} \in \mathcal{R}^{M}\right\} \\
& =\left\{\boldsymbol{w}_{O} \mid \boldsymbol{w}_{O}=\tilde{\boldsymbol{E}}_{P 2}^{T} \boldsymbol{x}, \boldsymbol{x} \in \mathcal{R}^{\bar{a}}\right\} .
\end{aligned}
$$

Then, the generalized force space of object can be spanned by the all columns of $\Xi$ and $\tilde{\boldsymbol{E}}_{P 2}^{T}$, namely rank $\left(\boldsymbol{\Xi} \tilde{\boldsymbol{E}}_{P 2}^{T}\right)=$ D.

Using this orthogonality, (21) and (22), respectively, are rewritten by

$$
\begin{gathered}
\text { DAFC }=\left\{\Delta r \mid \text { Work }=\Delta \boldsymbol{r}^{T} \boldsymbol{G}_{O J} \boldsymbol{\tau}_{c}>0, \Delta r \in \mathcal{A},\right. \\
\left(\boldsymbol{I}-\tilde{\boldsymbol{E}}_{P 2}^{T} \tilde{\boldsymbol{E}}_{P 2}\right) \boldsymbol{G}_{O J} \boldsymbol{\tau}_{c}+\boldsymbol{G}_{O}\left(\boldsymbol{I}-\left(\boldsymbol{J}^{T}\right)^{+} \boldsymbol{J}^{T}\right) \tilde{\boldsymbol{k}}_{1}=\boldsymbol{o}, \\
\left.\left(\boldsymbol{J}^{T}\right)^{+} \boldsymbol{\tau}_{c}+\left(\boldsymbol{I}-\left(\boldsymbol{J}^{T}\right)^{+} \boldsymbol{J}^{T}\right) \tilde{\boldsymbol{k}}_{1}+\boldsymbol{f}_{s t} \in \mathcal{F}_{f}\right\}, \\
\mathrm{DAFC}=\left\{\Delta \boldsymbol{r} \mid \Delta \boldsymbol{r}=\tilde{\boldsymbol{E}}_{P 2}^{T} \tilde{\boldsymbol{E}}_{P 2} \boldsymbol{G}_{O J} \boldsymbol{\tau}_{c}\right\} . \\
\text { VI. CONTROL LAW }
\end{gathered}
$$

\section{CONTROL LAW}

In this section, a control law is derived. In order to manipulate the object under the hybrid active/passive closure, we assume that the space spanned by the all columns of $\tilde{\boldsymbol{E}}_{P 2}^{T}$ is SAFC and that the space spanned by the all columns of $\boldsymbol{\Xi}$ is SPFC. Note that these assumptions correspond to assume a force-closure grasp, which is needed to grasp and manipulate the object stably.
A. Decomposition of object dynamics into the Parts corresponding to SAFC and SPFC

We decompose the object dynamics into the part corresponding to SAFC and the part corresponding to SPFC. By using the decomposition, we have only to control the part corresponding to SAFC because any applied force and moment in the direction contained in SPFC are counteracted without any additional joint torque input and then control is not needed (if the frictional constraints are satisfied).

From (4), (8), (13) and (18), the dynamics of the object is rewritten by

$$
\begin{array}{r}
M_{O r} \tilde{\boldsymbol{E}}_{P 2}^{T} \ddot{\bar{\zeta}}+\tilde{h}_{O r}=w_{O}=G_{O . J} \tau_{c}+\Xi k_{1}, \\
\tilde{h}_{O r}=h_{O r}+M_{O r} \dot{\tilde{E}}_{P 2}^{T} \dot{\tilde{\zeta}} .
\end{array}
$$

By multiplying $\tilde{\boldsymbol{E}}_{P 2}$ to the both side of (29) from the left side and using (27) (the orthogonality between SAFC (DAFC) and SPFC (DPFC)), we obtain

$$
\tilde{E}_{P 2} M_{O r} \tilde{E}_{P 2}^{T} \ddot{\tilde{\zeta}}+\tilde{E}_{P 2} \tilde{h}_{O r}=\tilde{E}_{P 2} G_{O J} \tau_{c} .
$$

This equation shows the component of object dynamics, which corresponds to SAFC. From the first assumption at the beginning of this section and (21') or (22'), $\tilde{\boldsymbol{E}}_{P 2} \boldsymbol{G}_{O J}$ has full row rank. Since $M_{O r}$ is nonsingular and $\tilde{\boldsymbol{E}}_{P 2}$ has full row rank, $\tilde{E}_{P 2} M_{O r} \tilde{E}_{P 2}^{T}$ is also nonsingular. Hence, an arbitrary acceleration in the direction contained in SAFC can be generated by the joint torques.

From (30), we obtain

$$
\tau_{c}=\left(\tilde{\boldsymbol{E}}_{P 2} G_{O J}\right)^{+}\left(\tilde{\boldsymbol{E}}_{P 2} \boldsymbol{M}_{O r} \tilde{\boldsymbol{E}}_{P 2}^{T} \ddot{\bar{\zeta}}+\tilde{\boldsymbol{E}}_{P 2} \tilde{\boldsymbol{h}}_{O r}\right)+\boldsymbol{\Phi} \boldsymbol{k}_{2} .
$$

where $\Phi \in \mathcal{R}^{M \times(M-\bar{a})}$ is a full column rank matrix whose columns form bases of the null space of $\tilde{\boldsymbol{E}}_{P 2} \boldsymbol{G}_{O J}$, and $\boldsymbol{k}_{2}$ $\in \mathcal{R}^{(M-\tilde{a})}$ is an arbitrary vector expressing the magnitude of each column of $\Phi$.

On the other hand, by multiplying $\Xi^{T}$ to the both side of (29) from the left side, the part corresponding to SPFC is given by

$$
k_{1}=\Xi^{T} M_{O r} \tilde{E}_{P 2}^{T} \ddot{\bar{\zeta}}+\Xi^{T} \tilde{h}_{O T}-\Xi^{T} G_{O J} \tau_{c} .
$$

Note that substituting $\tau_{c}$ given by (31) and $k_{1}$ given by (32) into the right side of (29) and using the relation; $\left(\Xi \tilde{E}_{P 2}^{T}\right)\left(\Xi \tilde{\boldsymbol{E}}_{P 2}^{T}\right)^{T}=\boldsymbol{I}$, we obtain $\boldsymbol{o}=\boldsymbol{o}$.

\section{B. Internal force}

From (8), the following relation is obtained;

$$
\tau_{c}=G_{O J}^{+}\left(w_{O}-\Xi k_{1}\right)+\Lambda \tilde{f}
$$

where $\Lambda \in \mathcal{R}^{M \times b}$ is a full column rank matrix whose columns consist of the bases contained in the space $\left(\operatorname{Im}\left(J^{T}\right) \cap \operatorname{ker}\left(G_{O J}\right)\right)$, and $\tilde{f} \in \mathcal{R}^{b}$ is an arbitrary vector expressing the magnitude of each column of $\Lambda$. Note that $\left(J^{T}\right)^{+} \boldsymbol{\Lambda} \tilde{f}$ is the internal forces which can be controlled by the joint torques, $\tau_{c}\left(G_{O}\left(J^{T}\right)+\Lambda \tilde{f}=o\right)$. Note also that since $\tau_{c}$ is contained in $\operatorname{Im}\left(J^{T}\right)$ from (6), $\Lambda$ must be contained in $\operatorname{Im}\left(J^{T}\right)$. 


\section{Dynamics of the total system}

From (4), (7), (12) and (18), the dynamics of the fingers is rewritten by;

$$
\begin{aligned}
\tau & =M_{q}\left(\tilde{\boldsymbol{E}}_{P 1}^{T} \ddot{\tilde{\zeta}}+\boldsymbol{E}_{P 11}^{T} \ddot{\hat{\zeta}}\right)+\tilde{h}_{q}+\tau_{c} \\
\tilde{h}_{q} & =M_{q}\left(\dot{\overline{\boldsymbol{E}}}_{P 1}^{T} \dot{\tilde{\zeta}}+\dot{\boldsymbol{E}}_{P 11}^{T} \dot{\hat{\boldsymbol{\zeta}}}\right)+h_{q} .
\end{aligned}
$$

Then, from (29), (33) and (34), the following relation is obtained;

$$
\begin{aligned}
\tau= & M_{q}\left(\tilde{E}_{P 1}^{T} \ddot{\bar{\zeta}}+E_{P 11}^{T} \ddot{\hat{\zeta}}\right)+\tilde{h}_{q}+\Lambda \tilde{\boldsymbol{f}} \\
& +G_{O J}^{+}\left(M_{O r} \tilde{E}_{P 2}^{T} \ddot{\bar{\zeta}}+\tilde{h}_{O r}-\Xi k_{1}\right)
\end{aligned}
$$

\section{Controller}

Assume that the desired trajectories $\tilde{\zeta}_{d}$ and $\tilde{f}_{d}$ for the movable object position/orientation $\bar{\zeta}$ and the internal force component $\tilde{f}$ are given. From (31), (33) and (34), the following linearizing control law is considered;

$$
\tau=M_{W} u_{p}+\Lambda u_{I}+Q+M_{q} E_{P 11}^{T} d_{\zeta d}
$$

where

$$
\begin{aligned}
M_{W} & =M_{q} \tilde{\boldsymbol{E}}_{P 1}^{T}+\left(\tilde{\boldsymbol{E}}_{P 2} \boldsymbol{G}_{O J}\right)^{+}\left(\tilde{\boldsymbol{E}}_{P 2} \boldsymbol{M}_{O r} \tilde{\boldsymbol{E}}_{P 2}^{T}\right), \\
\boldsymbol{Q} & =\tilde{\boldsymbol{h}}_{q}+\left(\tilde{\boldsymbol{E}}_{P 2} \boldsymbol{G}_{O J}\right)^{+}\left(\tilde{\boldsymbol{E}}_{P 2} \tilde{\boldsymbol{h}}_{O r}\right)
\end{aligned}
$$

and $u_{p}$ and $u_{I}$ are new control inputs and correspond to $\ddot{\bar{\zeta}}$ and $\tilde{f}$, respectively, and $d_{\zeta d}$ denotes an arbitrary constant vector that can provide finger motion that does not affect the object motion. $d_{\zeta d}$ is given such that the fingers meet a certain criterion.

By applying the control law to the system given by (35), we obtain

$$
\begin{aligned}
& M_{q} \tilde{E}_{P 1}^{T}\left(u_{p}-\ddot{\tilde{\zeta}}\right)+\Lambda\left(u_{I}-\tilde{f}\right)+M_{q} E_{P 11}^{T}\left(d_{\zeta d}-\ddot{\hat{\zeta}}\right) \\
& +\left(\tilde{E}_{P 2} G_{O J}\right)^{+}\left(\tilde{\boldsymbol{E}}_{P 2} M_{O r} \tilde{E}_{P 2}^{T} u_{p}+\tilde{E}_{P 2} \tilde{h}_{O r}\right) \\
& -G_{O J}^{+}\left(M_{O r} \overline{\boldsymbol{E}}_{P 2}^{T} \ddot{\bar{\zeta}}+\tilde{h}_{O r}-\Xi k_{1}\right)=\boldsymbol{o} .
\end{aligned}
$$

Here, since $\tilde{E}_{P 2} G_{O J}$ has full row rank, $\operatorname{Im}\left(\tilde{E}_{P 2}^{T}\right) \notin$ $\operatorname{ker}\left(\boldsymbol{G}_{O J}^{T}\right)$, namely $\operatorname{Im}\left(\tilde{\boldsymbol{E}}_{P_{2}}^{T}\right) \in \operatorname{Im}\left(\boldsymbol{G}_{O J}\right)$ and then $\left(G_{O J}^{+}\right)^{T} G_{O J}^{T} \quad \tilde{E}_{P 2}^{T}=\tilde{E}_{P 2}^{T^{2}}$. Hence, by multiplying $\tilde{E}_{P_{2}} G_{O J}$ to the both side of (37) from the left side and using the relation; $G_{O J} \Lambda=O, \tilde{E}_{P 2} G_{O J}\left(\tilde{E}_{P 2} G_{O J}\right)^{+}=$ $I, \tilde{E}_{P 2} G_{O J} G_{O J}^{+}=\tilde{E}_{P 2}$ and (27), the following relation is obtained;

$$
M_{w r}\left(u_{p}-\ddot{\tilde{\zeta}}\right)+M_{w r q}\left(d_{\zeta d}-\ddot{\hat{\zeta}}\right)=o
$$

where

$$
\begin{aligned}
M_{w r} & =\tilde{\boldsymbol{E}}_{P 2} M_{O r} \tilde{\boldsymbol{E}}_{P 2}^{T}+\tilde{\boldsymbol{E}}_{P 2} G_{O J} M_{q} \tilde{E}_{P 1}^{T}, \\
M_{w r q} & =\tilde{\boldsymbol{E}}_{P 2} G_{O J} M_{q} E_{P 11}^{T} .
\end{aligned}
$$

Since $\tilde{E}_{P 2} G_{O J}$ has full row rank, $\tilde{E}_{P 2} G_{O J}$ can be written by

$$
\left(\tilde{\boldsymbol{E}}_{P 2} \boldsymbol{G}_{O J}\right)^{+}=\boldsymbol{G}_{O J}^{T} \tilde{\boldsymbol{E}}_{P 2}^{T}\left(\tilde{\boldsymbol{E}}_{P 2} \boldsymbol{G}_{O J} \boldsymbol{G}_{O J}^{T} \tilde{\boldsymbol{E}}_{P 2}^{T}\right)^{-1} .
$$

From $\operatorname{Im}\left(\boldsymbol{E}_{P 11}^{T}\right) \in \operatorname{ker}(\boldsymbol{J}), \quad \boldsymbol{E}_{P 11}\left(\tilde{\boldsymbol{E}}_{P 2} \boldsymbol{G}_{O J}\right)^{+}=\boldsymbol{O}$. Hence, by multiplying $\boldsymbol{E}_{P 11}$ to the both side of (37) from the left side and using the relation; $E_{P 11} \Lambda=O$ (note that $\left.\operatorname{Im}(\Lambda) \in \operatorname{Im}\left(J^{T}\right)\right), \boldsymbol{E}_{P 11}\left(\tilde{\boldsymbol{E}}_{P 2} \boldsymbol{G}_{O J}\right)^{+}=\boldsymbol{O}$, and $E_{P 11} G_{O J}^{+}=O$, the following relation is obtained;

$$
M_{w q r}\left(u_{p}-\ddot{\bar{\zeta}}\right)+M_{w q}\left(d_{\zeta d}-\ddot{\tilde{\zeta}}\right)=o,
$$

where

$$
\begin{aligned}
M_{w q r} & =E_{P 11} M_{q} \tilde{E}_{P 1}^{T}, \\
M_{w q} & =E_{P 11} M_{q} E_{P 11}^{T} .
\end{aligned}
$$

Here, from (3) and (4), we obtain

$$
J E_{P 1}^{T} \dot{\zeta}=G_{O}^{T} E_{P 2}^{T} \dot{\zeta}
$$

From (18) and using the relation; $J E_{P 11}^{T}=O$, this equation is rewritten by

$$
J \tilde{E}_{P 1}^{T} \dot{\tilde{\zeta}}=G_{O}^{T} \tilde{E}_{P 2}^{T} \dot{\tilde{\zeta}}
$$

Since $\dot{\tilde{\zeta}}$ is arbitrary, we obtain

$$
J \tilde{E}_{P 1}^{T}=G_{O}^{T} \tilde{E}_{P 2}^{T} .
$$

By multiplying $J^{+}$to the both side of this equation from the left side and using the relation; $J^{+} J \tilde{E}_{P 1}^{T}=\tilde{E}_{P 1}^{T}$ (note that $\operatorname{Im}\left(\tilde{E}_{P 1}^{T}\right) \in \operatorname{Im}\left(J^{T}\right)$, we obtain

$$
\tilde{E}_{P 1}^{T}=G_{O J}^{T} \tilde{E}_{P 2}^{T}
$$

Then, $M_{w r}$ and $M_{w q r}$ become

$$
\begin{aligned}
M_{w r} & =\tilde{\boldsymbol{E}}_{P 2} M_{O r} \tilde{\boldsymbol{E}}_{P 2}^{T}+\tilde{\boldsymbol{E}}_{P 2} G_{O J} M_{q} G_{O J}^{T} \tilde{E}_{P 2}^{T} \\
M_{w q r} & =E_{P 11} M_{q} G_{O J}^{T} \tilde{E}_{P 2}^{T}=M_{w r q}^{T} .
\end{aligned}
$$

From (38) and (40), we obtain

$$
\left(\begin{array}{cc}
M_{w r} & M_{w r q} \\
M_{w r q}^{T} & M_{w q}
\end{array}\right)\left(\begin{array}{c}
\left(u_{p}-\ddot{\tilde{\zeta}}\right) \\
\left(d_{\zeta d}-\ddot{\tilde{\zeta}}\right)
\end{array}\right) \triangleq W\left(\begin{array}{c}
\left(u_{p}-\ddot{\tilde{\zeta}}\right) \\
\left(d_{\zeta d}-\ddot{\hat{\zeta}}\right)
\end{array}\right)=o .
$$

Since $M_{w q}$ is positive definite and then

$$
\begin{aligned}
W & =W_{b}\left(\begin{array}{cc}
W_{a} & O \\
O & M_{w q}
\end{array}\right) W_{b}^{T} \\
W_{a} & =M_{w r}-M_{w r q} M_{w q}^{-1} M_{w r q}^{T} \\
W_{b} & =\left(\begin{array}{cc}
I & M_{w r q} M_{w q}^{-1} \\
O & I
\end{array}\right),
\end{aligned}
$$

the determinant of $W$ is given by

$$
\operatorname{det} \boldsymbol{W}=\operatorname{det}\left(\boldsymbol{M}_{w q}\right) \operatorname{det}\left(\boldsymbol{W}_{a}\right) .
$$

Since $M_{q}$ is positive definite symmetric, we can define a nonsingular matrix $B_{m} \in \mathcal{R}^{M \times M}$ such that $M_{q}=\boldsymbol{B}_{m}^{T} \boldsymbol{B}_{m}$. Letting $\boldsymbol{B}_{r}^{T}=\tilde{\boldsymbol{E}}_{P 2} \boldsymbol{G}_{O J} \boldsymbol{B}_{m}^{T}$ and $\boldsymbol{B}_{q}^{T}=\boldsymbol{E}_{P 11} \boldsymbol{B}_{m}^{T}$, we obtain $\boldsymbol{W}_{a}=\tilde{\boldsymbol{E}}_{P 2} \boldsymbol{M}_{O r} \tilde{\boldsymbol{E}}_{P 2}^{T}+\boldsymbol{B}_{r}^{T}\left(\boldsymbol{I}-\boldsymbol{B}_{q}\left(\boldsymbol{B}_{q}^{T} \boldsymbol{B}_{q}\right)^{-1} \boldsymbol{B}_{q}^{T}\right) \boldsymbol{B}_{r}$ Since both $\boldsymbol{B}_{r}$ and $\boldsymbol{B}_{q}$ are of full rank, $\left(\boldsymbol{B}_{q}^{T} \boldsymbol{B}_{q}\right)^{-1} \boldsymbol{B}_{q}^{T}$ represents $B_{q}^{+}$. Then, the second term of this equation is nonnegative definite. Since $\tilde{E}_{P 2}$ is of full row rank, $\tilde{\boldsymbol{E}}_{P 2} M_{O r} \tilde{\boldsymbol{E}}_{P 2}^{T}$ is positive definite. Hence, $\boldsymbol{W}_{a}$ is positive 
definite. Therefore, from(42), $\operatorname{det} W \neq 0$. Thus, from (41), we obtain

$$
u_{p}=\ddot{\tilde{\zeta}}, \quad d_{\zeta d}=\ddot{\hat{\zeta}}
$$

From (39), $\boldsymbol{\Lambda}^{T}\left(\tilde{\boldsymbol{E}}_{P 2} \boldsymbol{G}_{O J}\right)^{+}=\boldsymbol{O}$. By multiplying $\boldsymbol{\Lambda}^{T}$ to the both side of (37) from the left side and using the relation; $\Lambda^{T}\left(\tilde{E}_{P 2} G_{O J}\right)^{+}=O$ and (43), the following relation is obtained;

$$
\boldsymbol{\Lambda}^{T} \boldsymbol{\Lambda}\left(u_{I}-\tilde{f}\right)=o .
$$

Since $\Lambda^{T} \boldsymbol{\Lambda}$ is nonsingular, we obtain

$$
u_{I}=\tilde{f}
$$

From (43) and (44), the system can be decoupled and linearized by the control law (36). Then, we adopt the following servo controllers with respect to $u_{p}$ and $u_{I}$;

$$
\begin{aligned}
& u_{p}=\ddot{\bar{\zeta}}_{d}+K_{V}\left(\dot{\bar{\zeta}}_{d}-\dot{\bar{\zeta}}\right)+K_{P}\left(\tilde{\zeta}_{d}-\tilde{\zeta}\right), \\
& u_{I}=\tilde{f}_{d}+K_{I} \int_{0}^{t}\left(\tilde{f}_{d}-\tilde{f}\right) d t^{\prime}
\end{aligned}
$$

where $K_{P}, K_{V}$ and $K_{I}$ are gain matrices. Then, the object position/orientation error $e_{p}=\tilde{\zeta}_{d}-\tilde{\zeta}$ and the force error $e_{f}=\tilde{f}_{d}-\tilde{f}$ satisfy the following equations;

$$
\begin{aligned}
\ddot{e}_{p}+K_{V} \dot{e}_{p}+K_{P} e_{p} & =o \\
\dot{e}_{f}+K_{I} e_{f} & =o
\end{aligned}
$$

Thus, with appropriate $\boldsymbol{K}_{\tilde{P}}, \boldsymbol{K}_{V}$ and $\boldsymbol{K}_{I}$, the actual object position/orientation $\tilde{\zeta}$ and the component of the internal forces $\tilde{f}$ will converge to the desired trajectories asymptotically.

\section{DETERMination of DESIREd INTERNAL ForCES}

In this section, it is shown how to determine the desired magnitudes of internal forces, $\tilde{f}_{d}$. These magnitudes are determined so that the frictional constraints can be satisfied during the manipulation.

At first, let us consider to formulate internal forces, with respect to contact forces, $f$. From (6), we get

$$
f=G_{O}^{+} w_{O}+\Omega k_{3}
$$

where $\Omega \in \mathcal{R}^{L D \times(L D-D)}$ is a full column rank matrix whose columns form bases of the null space of $G_{O}$ and $k_{3}$ $\in \mathcal{R}^{(L D-D)}$ is an arbitrary vector expressing the magnitude of each column of $\Omega$.

Here, $\boldsymbol{\Omega} \boldsymbol{k}_{3}$ expresses the internal forces. However, it is possible that $\Omega k_{3}$ includes the internal forces which cannot be controlled by the joint torques. Then, in (49), we use $\left(J^{T}\right)^{+} \boldsymbol{\Lambda} \bar{f}$, which is the internal forces that can be controlled by the joint torques, in place of $\Omega k_{3}$;

$$
f=G_{O}^{+} w_{o}+\left(J^{T}\right)^{+} \Lambda \tilde{f} .
$$

Then, assuming the object moves along the desired trajectory, we consider the following problem from (9), (29)

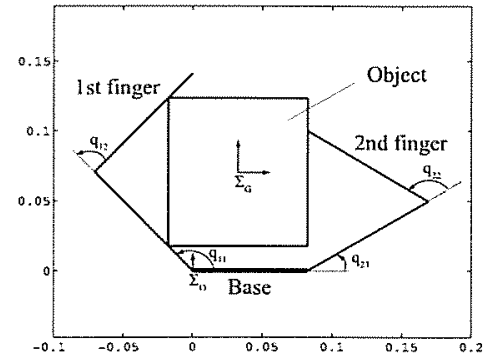

Fig. 2. Target System in Simulation (at the initial state)

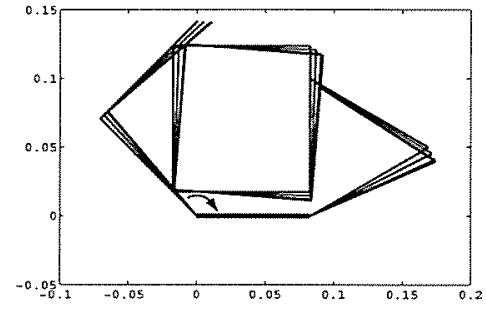

(a) Motion of the object

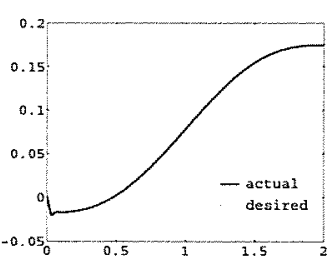

(b) Trajectory of $\phi$
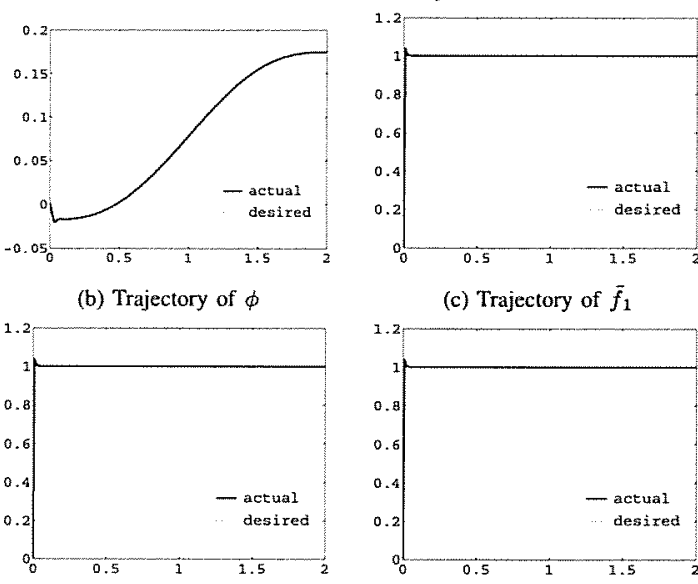

(d) Trajectory of $\tilde{f}_{2}$

(c) Trajectory of $\bar{f}_{1}$

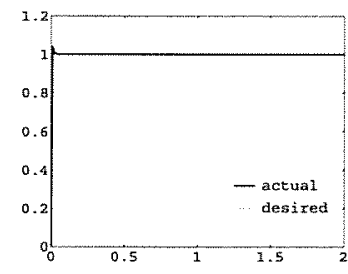

(e) Trajectory of $\tilde{f}_{3}$

Fig. 3. Results

and (50);

$$
\begin{aligned}
& \min _{\bar{f}} \quad \rho(\bar{f}) \\
& \text { subject to } f=G_{O}^{+}\left(w_{O_{d}}+w_{O_{\text {ext }}}\right)+\left(J^{T}\right)+\Lambda \tilde{f} \\
& \begin{array}{c}
f \in \mathcal{F}_{f} \\
w_{O_{d}}=M_{O r} \tilde{\boldsymbol{E}}_{P 2}^{T} \ddot{\boldsymbol{\zeta}}_{d}+\tilde{h}_{O r}
\end{array}
\end{aligned}
$$

where $\rho$ is a criterion function and $\boldsymbol{w}_{O_{e x t}}$ is unexpected external forces and moments. Note that for the practical use, we should better use smaller $\mu_{i j}$ in $\mathcal{F}_{f}$ than the real one. Note also that as a candidate for $\rho$, we can cite magnitudes of contact forces, magnitudes of joint torques, and so on.

\section{SIMULATION}

In order to verify our approach, we show simulation results. Fig. 2 shows the target system in the simulation. 
$\Sigma_{O}$ is placed at the root of the 1st finger. $\Sigma_{G}$ is placed at the geometrical center of the object. The scale markings shown in Fig. 2 represent the ones of $\Sigma_{R}$. Note that when the object is at the initial state, $\Sigma_{O}$ corresponds to $\Sigma_{R}$ (see Fig.2).

The robotic hand is composed of 2 fingers which are same form. The length of each link is set to $0.1[\mathrm{~m}]$. The mass of each link is set to $0.025[\mathrm{~kg}]$. The object is a $0.075 \sqrt{2} \times 0.1[\mathrm{~m}]$ quadrangle. The mass of the object is set to $0.408[\mathrm{~kg}]$. The density of the object is set to be uniform. The contact positions at the initial state are set to $\left(\begin{array}{lll}-0.0177 & 0.0177\end{array}\right),\left(\begin{array}{lll}-0.0177 & 0.1237\end{array}\right)$, and $\left(\begin{array}{lll}0.0823 & 0.1\end{array}\right)$. The servo gains are set to $K_{P}=\operatorname{diag}(10000.10000$. 10000.) [1/ $\left.\mathrm{sec}^{2}\right], K_{V}=\operatorname{diag}(100.100 .100).[1 / \mathrm{sec}]$, and $\boldsymbol{K}_{I}=\operatorname{diag}(100.100 .100$.) [1/sec]. The sampling time for calculating the input torques is set to $1.0[\mathrm{msec}]$ and the one for calculating the direct dynamics is set to $0.1[\mathrm{msec}$ ]

We consider to rotate counterclockwise the object around $\Sigma_{O}$ from 0 [degree] to 10 [degree]. However, to make an initial error, we rotate the object around $\Sigma_{O}$ from -1 [degree] to 10 [degree] in the desired trajectory. The frictional coefficients at all contact points are set to 0.3 . The desired magnitudes of internal forces are set to $\left(\begin{array}{lll}1 & 1 & 1\end{array}\right)[\mathrm{N}]$ which can satisfy the constraints of the problem given by (51).

Fig. 3 shows the results of the simulation. Fig.3 (a) shows the outline of the motion of the grasped object. Fig.3 (b) shows the trajectories of desired and actual $\phi$ 's that corresponds to the orientation of the object. Fig.3 (c) (e) show the trajectories of desired and actual $\tilde{f}_{i}$ 's $(i=1,2,3)$ that corresponds to the magnitudes of internal forces. From Fig.3, we can see that the $\phi$ and $\tilde{f}_{i}$ 's $(i=1,2,3)$ all converge to their desired trajectories, although there are initial errors.

\section{CONCLUSION}

In this paper, we have proposed a control algorithm for manipulating an object under hybrid active/passive closure. For the purpose, we have shown the orthogonality between the directions of active and passive force closures for general grasping systems. By using the orthogonality, we have decomposed the object dynamics into the part corresponding to active force closure and the part corresponding to passive force closure. By using the decomposition, we have shown that we have only to take into consideration the dynamics of the active part in order to derive a control algorithm. In order to satisfy the frictional constraints, we also have shown a way for determining desired internal forces. The simulation results show the effectiveness of our approach.

\section{REFERENCES}

11] A. Bicchi and V. Kumar, "Robotic grasping and contact: A review," Proc. of IEEE International Conference on Robotics and Automation, pp. 348-353, 2000.

[2] J. C. Trinkle, "On the stability and instantaneous velocity of grasped frictionless objects," IEEE Transactions on Robotics and Automation, vol. 8, no. 5, pp. 560-572, 1992.
[3] M. R. Cutkosky, "On grasp choice, grasp models, and the design of hands for manufacturing tasks," IEEE Transactions on Robotics and Automation, vol. 5, no. 3, pp. 269-279, 1989.

[4] T. Yoshikawa, "Passive and active closures by constraining mechanisms," Transaction of the ASME, Journal of Dynamic Systems, Measurement, and Control, vol. 121, pp. 418-424, 1999.

[5] T. Watanabe, Z. Jiang, and T. Yoshikawa, "Mechanics of hybrid active/passive-closure grasps." Proc. of IEEE International Conference on Robotics and Automation, pp. 1252-1257, 2004.

[6] T. Yoshikawa, Foundations of Robotics. MIT Press, Cambridge, 1990.

[7] Z. Li, P. Hsu, and S. Sastry, "Grasping and coordinated manipulation by a multifingered robot hand," The International Journal of Robotics Research, vol. 8, no. 4, pp. 33-50, 1989.

[8] A. B. A. Cole, J. E. Hauser, and S. S. Sastry, "Kinematics and control of multifingered hands with rolling contact," IEEE Transactions on Automatic Control, vol. 34, no. 4, pp. 398-403, 1989.

[9] Y. Yokokohji, M. Sakamoto, and T. Yoshikawa, "Vision-aided object manipulation by a multifingered hand with soft fingertips," Proc. of IEEE International Conference on Robotics and Automation, pp. 1027-1031, 1999.

[10] K. Harada, M. Kaneko, and T. Tsuji, "Active force closure for multiple objects," Journal of Robotic Systems, vol. 19, no. 3, pp. $133-141,2002$

[11] - "Rolling-based manipulation for multiple objects," IEEE Transactions on Robotics and Automation, vol. 16, no. 5, pp. 457 468, 2000.

[12] J. C. Trinkle and R. P. Paul, "Planning for dexterous manipulation with sliding contacts," The International Journal of Robotics Re search, vol. 9, no. 3, pp. 24-48, 1990.

[13] A. Bicchi, C. Melchiorri, and D. Balluchi, "On the mobility and manipulability of general multiple limb robots," IEEE Transaction on Robotics and Automation, vol. 11, no. 2, pp. 215-228, 1995.

[14] K. Harada and M. Kaneko, "A sufficient condition for manipulation of envelope family," IEEE Transactions on Robotics and Automation, vol. 18, no. 4, pp. 597-607, 2002.

[15] J. Park, W. Chung, and M. Kaneko, "Active-external enveloping grasps: Dynamical-balance based motion analysis," Proc. of the IEEE/RSJ International Conference on Intelligent Robots and Sys tems, pp. 1566-1571, 2001

[16] A. Shapiro, E. Rimon, and J. W. Burdick, "Passive force closure and its computation in compliant-rigid grasps," Proc. of the IEEE/RS International Conference on Intelligent Robots and Systems, pp. $1769-1775,2001$

[17] T. Omata and K. Nagata, "Rigid body analysis of the indeterminate grasp force in power grasp," Proc. of IEEE International Conference on Robotics and Automation, pp. 1787-1793, 1996.

[18] X.-Y. Zhang, Y. Nakamura, K. Goda, and K. Yoshimoto, "Robustness of power grasp," Proc. of IEEE International Conference on Robotics and Automation, pp. 2828-2835, 1994.

[19] K. Mirza and D. E. Orin, "General formulation for force distribution in power grasp," Proc. of IEEE International Conference on Robotics and Automation, pp. 880-887, 1994.

[20] Y. Zhang and W. A. Gruver, "Definition and force distribution of power grasps," Proc. of IEEE International Conference on Robotics and Automation, pp. 1373-1378, 1995.

[21] Y. Yu, K. Takeuchi, and T. Yoshikawa, "Optimization of robot hand power grasps," Proc. of IEEE International Conference on Robotics and Automation, pp. 3341-3347, 1998.

[22] T. Omata, "Rigid body analysis of power grasps: Bound of the indeterminate grasp force," Proc. of IEEE International Conference on Robotics and Automation, pp. 2203-2209, 2001.

[23] T. Yoshikawa, T. Watanabe, and M. Daito, "Optimization of power grasps for multiple objects," Proc. of IEEE International Conference on Robotics and Automation, pp. 1786-1791, 2001.

[24] M. Y. Wang and D. M. Pelinescu, "Contact force prediction and force closure analysis of a fixtured rigid workpiece with friction," Transactions on ASME, Journal of Manufacturing Science and Engineering, vol. 125 , pp. $325-332,2003$.

[25] T. Watanabe and T. Yoshikawa, "Optimization of grasping an object by using required acceleration and equilibrium-force sets," Proc. of IEEE/ASME Intemational Conference on Advanced Intelligen Mechatronics, pp. 338-343, 2003. 